\title{
A Review on Active Ingredients from Marine Sources used in Cosmetics
}

\author{
Latha Uppala* \\ Department of Pharmaceutics, SSJ College of Pharmacy, Hyderabad, India
}

Received: October 17, 2015; Accepted: November 4, 2015; Published: December 3, 2015

*Corresponding author: Latha Uppala, Department of Pharmaceutics, SSJ College of Pharmacy, Hyderabad, India, E-mail: chowdarylatha79@gmail.com

\section{Introduction}

The word cosmetic represents a specific product containing active ingredients. These cosmetics products with biologically active ingredients purporting to have medical benefit. They are formulated not only to improve the skin appearance but also to improve positive physiological effects at the cellular level. Simultaneously, it is necessary to discover new bioactive substances with resourceful, safe and stable properties from natural sources for cosmetic development. Recently marine resources have been demonstrated with great cosmetic prospective. It is a productive source of cosmetics.

Various compounds secluded from marine organisms show various cosmetic activities such as antioxidant, anti inflammatory, anti allergic, anti aging and anti wrinkling effects, tyrsinase and metalloproteinase inhibition and ultra violet defense. Cosmetic products formulated with sea water and sea creatures, such as marine collagen, astaxanthin, caviar and pearls etc. Cosmetic compounds derived from marine sources having activities with health benefits. It consists of a large number of phytochemical components mutual together that has been isolated from various marine resources.

\section{Keywords}

Cosmetics; Marine cosmetics; Skin health; Skin care.

\section{Definition}

When cosmetics contain an active ingredient from marine sources is known as marine cosmetics.

\section{History of marine cosmetics}

The earth consists of 70\% water, so it comes as no revelation that manufacturers are searching for new sources of ingredients from oceans and fresh water sources. Oceans have borne most of the biological activities on our planet. The ocean is the mother of life and it is held that the most prehistoric forms of life originated from the "primordial soup" and harbors a huge variety of marine organisms that are varied in their physiology and adaptations.

Marine organisms form a stupendous component of the oceanic population, which considerably contribute in the protection of cosmetics and pharmaceutical molecules with biologically accomplished moieties.

In today's feisty landscape, researchers are focusing on a new generation of high tech cosmetics that utilize ingredients sourced from the ocean.

Bioactive substances derived from marine resources have various useful roles as natural skin care agents, and these properties can be applied to the improvement of new cosmetics as well as nutricosmetics (from edible seaweeds and edible marine animals). This association focuses on marine-derived cosmetics active ingredients and presents a sign of their health beneficial effects on human skin.

Recently, generous studies have been conducted on the general aspects of the chemical structures, physical and biochemical properties and biotechnological applications of bioactive substances derived from marine organisms. In this review, we have discussed recent progresses in the biotechnological applications of bioactive substances from marine organisms as cosmetics.

Some Active Ingredients from marine sources used in cosmetics:

1. Marine Sponges

2. Seaweed

3. Shark fish

4. Marine turtles

5. Coral

6. Jelly fish

7. Hydras

8. Phytoplankton

9. Sea fennel... etc

Marine Sponges: The sea sponge is a renewable natural resource. The sea sponges are highly absorbent, create a luxurious lather, are softly textured and are suitable for the most insightful skin. Natural sponges are stronger and last longer than 
synthetic sponges because they are more resistant to abrasion.

Natural sea sponges actually have enzymes in them but impede the growth of mold, mildew and bacteria. In addition natural sea sponges are enhanced for bathing and cleaning because they soak up and hold more water without drenched. Mainly sponges are used to clean body and face where as mainly these are used for babies to clean their insightful skin. And also used as skin whitening compound.

Seaweed: Seaweed is also known as brown seaweed, red seaweed, algae, kelp, egg wrack, kombu/konbu, sea spaghetti, wakame, nori, dulse/dillisk, sea lettuce, sea grass, carrageenin, and Irish moss

Seaweed has been used in bath, body and skincare products for many centuries to improve circulation and balance natural moisture levels. This can help reinstate the texture and tone of the skin and reduce the appearance of cellulite. Historically Seaweed has been used for its detoxifying properties and as an aid to skin healing. Seaweed is rich in mineral salts, amino acids and Vitamins A, C, B1, B12, E, K and D. The lipid, protein, mineral and vitamin content are easy to absorb and present a richly hydrating and skin fostering treatment.

The main use of seaweed extract is for skin and body care it acts as Skin Health Protection, Skin Whitening, anti acne, anti ageing. It can rouse renewal of damaged skin cells. It can detoxify and cleanse, tone the skin and moisturizing.

Shark fish: Shark fish liver oil is highly rich in omega 3 fatty acids, other labeled squalenes or squalane, found in many products together with, moisturizers, deodorants, sun tan lotion, lip balms, lipsticks, perfumes, shampoos and other cosmetics. Many commercially formed brands of moisturizer, sunscreen, lipstick and eye makeup contain a compound derived from shark liver oil known as "squalene". The oily organic compound (along with squalane, a derivative) is a favored addition to many beauty products because of its moisturizing properties.

Marine Turtles: There is a suggestion that turtle oil had long been used for skin care by the original inhabitants of the Americas, it did not find its way into Western skin creams, in any major way, until the 1930s. The practice seems to have started in Europe and maybe came out of the same German and French research into the use of animal serums and extracts - opotherapy/ organotherapy - that lead to the introduction of hormones and other tissue extracts in skin creams.

Mainly turtle oil is extracted by heating the fat and the oil is extracted which is being used in the preparation of cosmetics, where the oil is rich in Vit-E. In England it is being started over there in cosmetic preparations like in bathing soaps, lotions, skins creams and nail creams, etc

Coral: Coral powders have been used in numerous cosmetic products, being touted as copious and unique. It has been used to scrub skin and supplying trace minerals to it. The prospective for using fossilized coral powder as a new sustainable material for cosmetic applications supported by its physical, chemical and textural characteristics, as well as its trace mineral content. Chemically coral powder is a pure material, composed mostly of calcium carbonate. However, this natural marine coral powder may contain 74 additional trace minerals, with absence of heavy metals. This coral powder is used to protect from UV radiation and acts as anti oxidant, anti ageing and anti acne.

It is used to smoothen the skin, in lipstick preparations, powders and deodorants.

Jelly fish: The mucus formed by jellyfish is rich $\mathrm{n}$ a compound that is vital for some cosmetics and drugs. According to Cosmetic Design, the cosmetic industry can step in and help to increase the fish stock by using jellyfish in the manufacture of anti-aging beauty products. Jellyfish have powerful anti-aging properties. Scientist have replicated the cells from the jelly fish within the peptide, juvefoxo and combined within skincare creams, it works to treat and prevent DNA damage and persuade our skin cells to act young again and regenerate.

Hydras: Hydras mainly consist of a gel which is used in all preparations which consists of antioxidants and proteins. Mainly hydras are used in preparation of lipsticks, lip gloss and many other products related to lips.

And also used to clean skin and used as a makeup remover without any reaction and also as moisturizer. It contains Vitamin- $C$ and Vitamin-E which is used in sunscreen lotions to protect from UV radiation and moisturizing cream. It is rich in olive oil with emollient properties.

Phytoplankton: Phytoplankton Extract, rich in lipids and omega-3 fatty acids stimulates the cells to produce cerebrosides (pro-ceramides), strengthen cellular cohesion, renews the skin's protective barrier. Mainly used for skin toning, skin whitening, anti wrinkling and ageing etc.

Sea Fennel: Sea fennel contains the antioxidant and antiaging trifecta (vitamins $\mathrm{A}, \mathrm{C}$ and $\mathrm{E}$ ) i.e. proven to prevent wrinkles and skin stressors brought on by modern-day life (like UV light, alcohol pollution, and cigarette smoke). "It also has chlorogenic acid, which is an effective antioxidant," making this subtle seaside plants are powerful skin protector. The potent stem cells also regulate the regeneration of keratin in your own skin which will improve the tone of skin

\section{Advantages in marine cosmetics}

- $\quad$ Marine ingredients are picking up steam in skin care, as they offer a variety of benefits, more advanced scientifically and eco-friendly.

- Marine ingredients can help provide vitamins and minerals to skin, UV and antioxidant protection, anti-aging benefits and more.

- $\quad$ The harvesting and preserving of marine ingredients has become more intricate over the past several decades, helping to make more effective as well as more environment friendly ingredients.

- The human body needs substitute of materials such 
as zinc, phosphorous, calcium and magnesium on a daily basis because sea water contains the body's idyllic balance of minerals.

- The marine drug is rich source of biological and chemical diversity.

- Marine ingredients have anti oxidant properties that have been used in skin-care products to prevent or even restore the damage caused by environmental factors, such as UV rays and low humidity, as well as damage allied with the aging process.

- Marine consequent proteins which can provide equivalents to collagen and gelatin without the associated risks are becoming more popular among consumers because of their abundant health beneficial effects.

- Most marine bioactive peptides are currently underutilized. While fish and shellfish are possibly the most evident sources of such proteins and peptides, there is also scope for further development of proteins and peptides from source like algae, sea cucumber and mollusks.

- The anti-oxidant properties help the skin cells in fighting all the free radicals that are produced as a result of radiation and chemical exposure to sun.

- It helps in restoring the normal hydration of skin cells that prevent the signs of aging and protect against wrinkle formation.

- $\quad$ Promotes natural cleansing of skin by opening pores; there by helps in restoring shining clear skin besides rehydration and regeneration skin cells.

\section{Conclusion}

Marine organisms produce unique compounds. Cosmetics derived from marine sources having health benefits for body. It consists of large number of phytochemical components combined together that have been secluded from various marine resources the current progress opens an opportunity towards understanding marine natural products and their potential for cosmetic purpose. For some years, marine-based ingredients have been sources of fascination for cosmetic companies.

\section{References}

1. Michael A. Borowitzka. Microalgae as sources of pharmaceuticals and other biologically active compounds. Journal of Applied Phycology. 1995;7(1):3-15. DOI: 10.1007/BF00003544.

2. Anna Hunter. Seaweed: The super food for skin, hair and health. 2013.

3. Jared Paventi. What Are the Benefits of Seaweed for Skin? Live strong. 2013.

4. Wijesinghe W.A.J.P,You-Jin Jeon. Biological activities and potential cosmeceutical applications of bioactive components from brown seaweeds: a review. Phytochemistry Reviews2011;10(3):431-443.

5. Babitha S, Kim E.K. Effect of marine Cosmeceuticals on the Pigmentation of Skin. In:Kim s.k.,editor.Marine Cosmeceuticals:Trends and Prospects. CRC Pres;Boca Ranton. 2011:63-66.

6. Pallela R, Na-Young Y,Kim SK. Anti-Photoaging and photoprotective compounds derived from marine organisms.Mar. Drugs.2010;8(4):1189-1202. doi:10.3390/md8041189

7. Elena M. Balboa, Enma Conde, M. Luisa Soto, Lorena PérezArmada, Herminia Domínguez. Cosmetics from Marine Sources. Springer Handbook of Marine Biotechnology. 2015;1015-1042. DOI: 10.1007/978-3-642-53971-8_44.

8. Dhabarde DM, Potins VV, Kamble MA, Ingole AR, Sant AP. Marine Cosmeceuticals. UJP. 2013;2(2):20-22. 